\title{
Visuospatial Perceptual Sequence Learning and Eye Movements
}

\author{
Daphné Coomans, Natacha Deroost, Jochen Vandenbossche, \\ Eva Van den Bussche, and Eric Soetens
}

\author{
Department of Cognitive Psychology, Vrije Universiteit Brussel, Belgium
}

\begin{abstract}
We examined perceptual sequence learning by means of an adapted serial reaction time task in which eye movements were unnecessary for performing the sequence learning task. Participants had to respond to the identity of a target letter pair ("OX" or "XO") appearing in one of four locations. On the other locations, similar distractor letter pairs ("QY" or "YQ") were shown. While target identity changed randomly, target location was structured according to a deterministic sequence. To render eye movements superfluous, (1) stimulus letter pairs appeared around a fixation cross with a visual angle of $0.63^{\circ}$, which means that they appeared within the foveal visual area and (2) the letter pairs were presented for only $100 \mathrm{~ms}$, a period too short to allow proper eye movements. Reliable sequence knowledge was acquired under these conditions, as responses were both slower and less accurate when the trained sequence was replaced by an untrained sequence. These results support the notion that perceptual sequence learning can be based on shifts of attention without overt oculomotor movements.
\end{abstract}

Keywords: implicit learning, perceptual sequence learning, eye movements, attention shifts

Acquiring knowledge about regularities in the environment, or sequence learning, is a fundamental human ability. The fact that it is already observed early in childhood (e.g., Clohessy, Posner, \& Rothbart, 2001) and that it can occur implicitly, that is, without the intention to learn and with the obtained knowledge being difficult to verbalize (Cleeremans, Destrebecqz, \& Boyer, 1998; Clegg, DiGirolamo, \& Keele, 1998), underlines that sequence learning is a powerful learning tool. In the current research, we examined whether sequence learning can occur when eye movements are unnecessary for performing the sequence learning task.

Implicit sequence learning is typically investigated with a serial reaction time task (SRT task), in which participants react to the location of a target on the screen with a spatially corresponding key. Without informing them, a sequence is imposed on the target location. Two main results indicate learning: (1) a decrease in error rates/reaction times (RTs) as the training progresses, which demonstrates a general training effect, and (2) higher error rates and/or slower responses when the trained sequence is replaced by an untrained sequence, which is indicative for sequence-specific learning.

Learning in the SRT task has been shown to rely on different kinds of knowledge. First, it can be based on motor knowledge or knowledge about the response movements made to the target, also called R-R learning (e.g., Deroost \& Soetens, 2006a; Nattkemper \& Prinz, 1997; Willingham, 1999; Willingham, Nissen, \& Bullemer, 1989). Secondly, it can rely on perceptual knowledge or knowledge about the visuospatial shifts of the stimulus, also called S-S learning (Coomans, Deroost, Zeischka, \& Soetens, 2011; Deroost \& Soetens, 2006b; Remillard, 2003, 2009, 2011). In addition, the S-R account stresses the importance of knowledge about the rules by which stimuli and responses are associated (Deroost \& Soetens, 2006c; Schumacher \& Schwarb, 2009; Schwarb \& Schumacher, 2009, 2010). Finally, a last account proposes that sequence knowledge is based on associations between responses and stimuli or effects, referred to as R-S learning (Ziessler, 1998; Ziessler \& Nattkemper, 2001).

In contrast to motor learning and related accounts in which motor responses are involved (S-R and R-S account), the existence of perceptual learning, or S-S learning, has proven very hard to demonstrate with the SRT task. Several researchers have been able to report null effects in this respect by showing that perceptual sequence learning was absent in their experiments (e.g., Kelly \& Burton, 2001; Rüsseler, Münte, \& Rösler, 2002; Willingham, 1999; Willingham et al., 1989). For instance, Willingham (1999) found that simply observing a sequence without responding to it does not lead to implicit sequence learning, an observation which casted doubt on the very existence of perceptual sequence learning.

Yet, a few authors were able to observe reliable perceptual learning. In the study of Mayr (1996), for example, participants responded to the identity of a target that appeared in one of four locations. The relevant target identity and the irrelevant target location were both structured according to two uncorrelated sequences. Mayr found that, although the location sequence was not responded to, both sequences were learned by the participants. However, target locations in this study were widely separated $\left(24^{\circ}\right.$ visual angle), so that participants were obliged to make overt eye movements in order to locate the target. Mayr therefore acknowledged that learning in his paradigm might reflect learning of a sequence of 
oculomotor movements, instead of learning of a sequence of attention shifts or perceptual learning (see also Willingham, 1999 for a similar remark). In line with this notion, oculomotor learning in the SRT task has been convincingly demonstrated in a number of studies. For example, Kinder, Rolfs, and Kliegl (2008) asked participants to respond to the target by simply looking at a dot that appeared in one of four locations. The sequence of target locations was structured. The authors found that participants' saccades were slower in a block that did not follow the learned sequence than in the adjacent structured blocks, which supports oculomotor sequence learning (see also Albouy et al., 2006; Marcus, Karatekin, \& Markiewicz, 2006).

Importantly, since the precise relationship between eye movements and attention shifts is still the subject of discussion, it remains unclear whether perceptual sequence learning can also occur without overt eye movements. Some attention theories presume that overt eye movements are always preceded by attention shifts (e.g., Godijn \& Pratt, 2002; Posner, 1980) and that the system responsible for the planning of oculomotor movements operates independently from the system responsible for the planning of attention shifts (e.g., Abrams \& Pratt, 2000; Posner, 1980). Based on these theoretical assumptions, perceptual learning might be based on attention shifts without the involvement of the oculomotor system (Remillard, 2003). In contrast, the premotor theory of attention of Rizzolatti and colleagues (Rizzolatti, Riggio, Dascola, \& Umiltà, 1987) proposes that both overt and covert attention shifts are a consequence of (programming) an eye movement. Based on this theory, Kinder and colleagues (2008) argued that perceptual sequence learning, or learning of a visuospatial sequence of target locations, can be the result of oculomotor learning.

To this day, the exact contribution of overt eye movements to perceptual sequence learning remains to be determined. Studies that reported perceptual sequence learning without a concurrent sequenced response dimension are scarce, and only one study attempted to control explicitly for eye movements. More particularly, Remillard (2003, Experiment 3) manipulated the visual angle of target locations to investigate the role of eye movements. In this experiment, six horizontally displayed pairs of vertical lines (“||" or " "l,") were presented with the distance between the leftmost and the rightmost location measuring only $3.9^{\circ}$. After $400 \mathrm{~ms}$, one of these letter pairs was marked and participants had to react to the identity of the marked letter pair ("I|" required a left response, "|l," a right one). While target identity always varied randomly, target location was sequenced. Importantly, sequence learning observed in this experiment was very similar to learning observed using a larger visual angle of $14^{\circ}$ (Experiment 1$)$. This seems to suggest that eye movements do not play a crucial role in perceptual sequence learning. Nonetheless, although the visual angle was reduced in Remillard's experiment, letter pairs were still placed horizontally with a width of $3.9^{\circ}$ visual angle, which means that eye movements were still necessary to locate the target.

A stronger argument against the necessity of eye movements in perceptual learning of a visuospatial sequence would be a demonstration of sequence learning when overt eye movements are unnecessary for performing the learning task. This was precisely the aim of the current study, in which we investigated perceptual sequence learning in an adapted version of the paradigm used by Remillard (2003). Participants were instructed to react to the identity of a target letter pair ("XO" or "OX") presented in one of four locations. The response dimension, that is, target identity, changed randomly, while the irrelevant location of the target was structured. On the three remaining locations distractor letter pairs were shown ("YQ" and "QY") that shared low-level features with the target letter pair. The distractor letter pairs were presented in order to guarantee optimal conditions to observe learning. In an earlier study (Coomans et al., 2011), perceptual sequence learning of horizontal target locations was shown to occur independently of perceptual load, but was only expressed when distractors were present. Like most studies on perceptual sequence learning, however, this study did not control for eye movements, as locations were separated by $3.43^{\circ}$ visual angle. This means that, like in Remillard's study (2003), eye movements were required to perform the task. This problem was addressed in the current study.

In order to render eye movements unnecessary, two important adaptations were made to the paradigm. First, the letter pairs were presented in a circular display with a small visual angle. As the distance from the fixation cross to the letter pairs was substantially less than $1^{\circ}$ visual angle, eye movements were no longer needed to locate the target (see Eriksen \& St. James, 1986). Second, we drastically limited the stimulus duration, by presenting stimuli for only $100 \mathrm{~ms}$. It is generally agreed that oculomotor movements require at least $150-175 \mathrm{~ms}$, so a stimulus duration of $100 \mathrm{~ms}$ is too short to make a saccade (Rayner, 1998). If perceptual sequence learning of a visuospatial sequence does not rely on overt eye movements, as proposed by Remillard (2003), then perceptual learning should occur using this paradigm in which eye movements are unnecessary. If, however, perceptual learning is merely a by-product of eye movements, as postulated by Kinder et al. (2008), then learning should not be observed.

\section{Method}

\section{Participants}

Forty-five students of the Vrije Universiteit Brussel (13 men, mean age $=19.29, S D=1.80$ ) executed the experiment in return for course credit of an introductory psychology course.

\section{Stimuli and Apparatus}

The experiment was programmed in E-Prime version 2.0 (Psychology Software Tools; Schneider, Eschman, \& Zuccolotto, 2002) and run on Pentium four personal computers with 17-inch CRT monitors. Participants completed the experiment individually in semidarkened cubicles of the psychological laboratory of the Vrije Universiteit Brussel. 
A black fixation cross $(0.2 \mathrm{~cm} \times 0.2 \mathrm{~cm})$ appeared in the center of the screen. Around the fixation cross (above, below, left, or right), one target letter pair ("XO" or "OX") was presented. On the remaining three locations, distractor letter pairs ("YQ" and "QY") were displayed. The letter pairs were depicted in Arial point size 6 , measured $0.5 \mathrm{~cm}$ width $\times 0.3 \mathrm{~cm}$ height, and were presented in black against a white background. The distance between the center of the fixation cross and the center of the letter pairs was $0.6 \mathrm{~cm}$ or $0.63^{\circ}$ at a viewing distance of approximately $55 \mathrm{~cm}$.

\section{Procedure}

Participants were urged to focus on the fixation cross during the experiment. Both target and distractors were presented for $100 \mathrm{~ms}$, after which only the fixation cross remained on the screen. Participants were asked to respond as fast and accurately as possible to the identity of the target letter pair. The target letter pair "XO" required a "C" response with the left index finger, the letter pair "OX" required an "N" response with the right index finger. After a correct response, the next trial started after a response-stimulus interval of $400 \mathrm{~ms}$. Four hundred milliseconds after an incorrect response, the word "error" in Dutch appeared on the screen for $750 \mathrm{~ms}$. After the offset of the error message, the next trial was presented. Finally, if no response was detected within 3,000 $\mathrm{ms}$, an error message was displayed.

Participants first completed two practice blocks, which consisted of 50 trials each. In these blocks, the location of the target varied pseudorandomly, with location repetitions being excluded. Target and distractor identities always varied randomly and individually per trial, with an equal probability of each target letter pair ("XO" or "OX") and distractor letter pair ("YQ" or "QY") to be presented. After each block, participants received feedback about their error rates and RTs, followed by a rest break of $30 \mathrm{~s}$.

Following the practice blocks, 15 experimental blocks of 100 trials each were presented. Unknown to the participants, target location in these blocks was structured. Two deterministic eight-element sequences (adopted from Albouy et al., 2006) were used, that is, 42132431 (S1) and 13423124 (S2), with the numbers referring to the four locations ( 1 for the location above the fixation cross and the rest of the numbers referring to the locations in a clockwise manner). The two sequences were characterized by first-order restrictions and were structurally identical. Participants were trained on S1 (or S2) in all blocks but test Block 13. In test Block 13, the alternate sequence S2 (or S1) was imposed on the target location. The replacement of the trained sequence by the untrained sequence in test Block 13 was done to establish sequence-specific learning of the trained sequence.

\section{Results}

The overall mean error rate was $12.23 \%(S D=7.85)$. This is quite high, which is not surprising as the task was very difficult to perform due to the brief stimulus presentation of $100 \mathrm{~ms}$. Three participants whose average error rates exceeded the mean plus two standard deviations were discarded from further analyses. For the RT analyses, mean median RTs from correct responses were used.

\section{General Training Effect}

First, we determined whether RTs and error rates decreased during the training, which would point to a general training effect. A repeated-measures ANOVA with Blocks 1-12 as within-subjects factor was performed. When the sphericity assumption was not met, the Greenhouse-Geisser correction was reported. Figure 1 depicts the mean median RT (Figure 1a) and the mean error rate (Figure 1b) per block.

\section{Reaction Times}

The RT analysis demonstrated a significant effect of Block, $F(2.99,122.38)=43.72, M S E=12978, p<.001$. A significant linear trend, which can be observed by calculating polynomial contrasts, showed that RTs decreased linearly
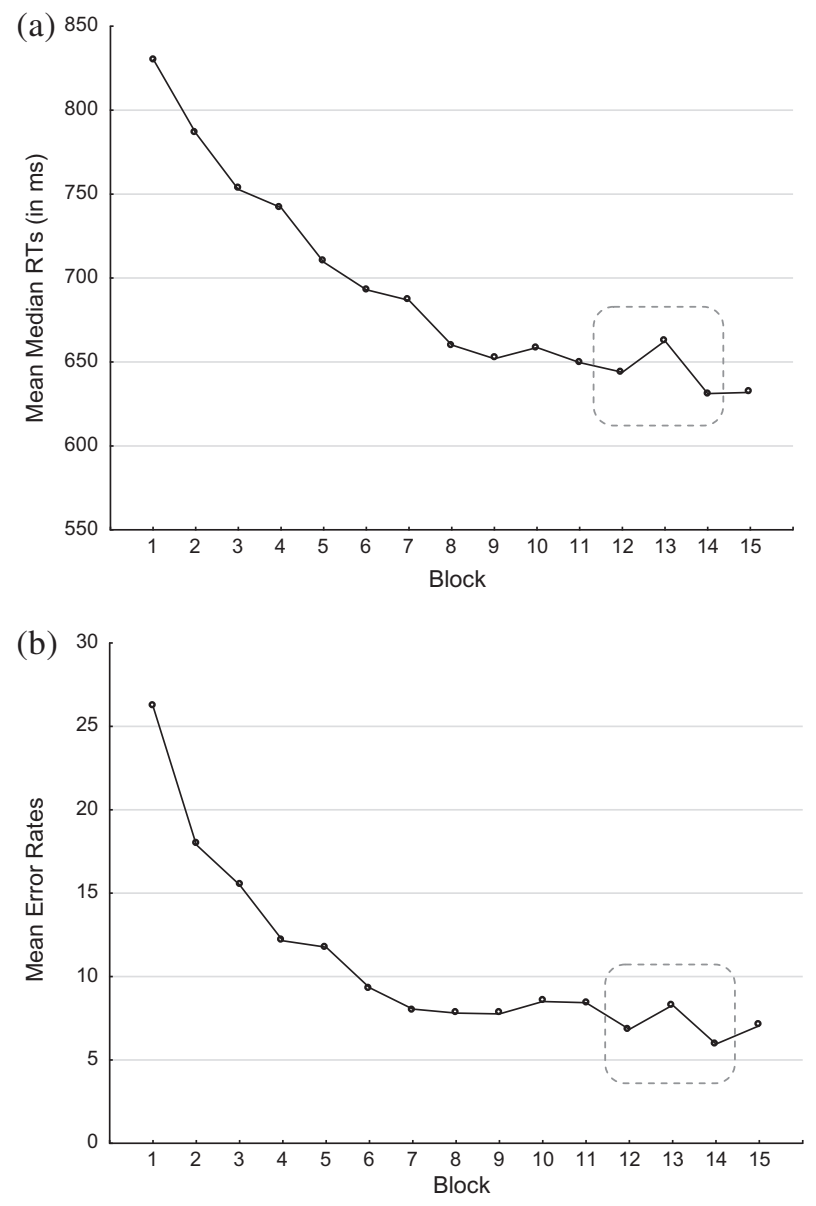

Figure 1. (a) Mean median reaction times and (b) mean error rates as a function of Block. In test Block 13, the trained sequence was replaced by an untrained sequence. 
during the training phase, $F(1,41)=107.90, M S E=14077$, $p<.001$.

\section{Error Rates}

The ANOVA showed that a significant effect of Block was present in the error rates, $F(3.24,132.70)=46.44, M S E=$ $100, p<.001$. Error rates dropped linearly during the first 12 blocks, as apparent from a linear trend in the ANOVA, $F(1,41)=89.36, M S E=120, p<.001$.

\section{Sequence-Specific Learning}

To assess learning of the trained sequence, the average of the RTs and the error rates in Block 12 and Block 14 was calculated. This average was subsequently compared to the RTs and error rates in test Block 13, in which the trained sequence was replaced by an untrained sequence, using paired samples $t$-tests. Slower RTs and/or higher error rates in test Block 13 than in the surrounding sequenced blocks imply that sequence-specific learning has taken place.

\section{Reaction Times}

A paired samples $t$-test showed that RTs in test Block 13 were higher than RTs in Blocks $12-14, t(41)=3.77$, $p<.001$, which indicates that participants learned the perceptual location sequence (see Figure 1a). RTs in Block 13 ran up to $663 \mathrm{~ms}$ and the average RT of Blocks 12 and 14 amounted to $638 \mathrm{~ms}$, which resulted in a learning effect of $25 \mathrm{~ms}[S D=43.1]$.

\section{Error Rates}

A paired samples $t$-test showed that participants made more errors in test Block 13 than in the surrounding trained sequenced Blocks 12 and 14, $t(41)=3.03, p<.01$ (see Figure 1b). The average learning effect measured $1.89 \%$ $[S D=4.05 ; 8.29 \%$ (Block 13) vs. $6.39 \%$ (Blocks 12-14) $]$.

\section{Motor Effects}

Our results show that perceptual learning can occur when oculomotor movements are unnecessary. However, because our perceptual learning effect is still indirectly derived from manual responses given to a motor dimension (stimulus identity) that varied independently from the perceptual dimension, we determined whether manual motor effects might have contributed to our observations.

\section{Response Repetition Effects}

First, we examined whether response repetition effects changed as a function of sequence learning. To this end, responses on response repetition and response alternation trials in Block 13 (untrained sequence) and Blocks 12-14 (trained sequence) were analyzed.

\section{Reaction Times}

Median RTs of correct responses were analyzed. The repeated-measures ANOVA with Repetition (response repetition vs. response alternation) and Sequence learning (Block 13 vs. the average of Blocks 12 and 14) as within-subjects factors revealed no main effect of Repetition, $F(1,41)=$ $1.26, M S E=887, p=.27$. As expected, a main effect of Sequence learning was present, $F(1,41)=18.15, M S E=$ $1739, p<.001$. Interestingly, there was no interaction between Repetition and Sequence learning, $F(1,41)<1$, indicating that there was equal sequence learning displayed in response repetition trials and in response alternation trials $\left[M_{\text {rep }}=659 \mathrm{~ms}\right.$ (Block 13) and $634 \mathrm{~ms}$ (Blocks 12-14), $M_{\text {alt }}$ $=666 \mathrm{~ms}$ (Block 13) and $637 \mathrm{~ms}$ (Blocks 12-14)].

\section{Error Rates}

The error rate analysis confirmed the results found in the RT analysis. The repeated-measures ANOVA showed no main effect of Repetition, $F(1,41)<1$, but a main effect of Sequence learning was present, $F(1,41)=9.33, M S E=$ $3.93, p<.01$. Finally, the Repetition $\times$ Sequence learning interaction was not significant, $F(1,41)<1\left[M_{\text {rep }}=\right.$ $4.10 \%$ (Block 13) and 3.19\% (Blocks 12-14), $M_{\text {alt }}=$ $3.88 \%$ (Block 13) and 2.92\% ms (Blocks 12-14)].

\section{Compatibility Effects}

We also investigated possible compatibility effects between stimulus location and response location. More specifically, we determined differences between stimulus-response compatible trials (e.g., stimulus XO, requiring a left response, appearing at the location left from the cross, or stimulus $\mathrm{OX}$, requiring a right response, appearing at the location right from the cross) and stimulus-response incompatible trials (stimulus OX, requiring a right response, appearing at the location left from the cross, or stimulus XO, requiring a left response, appearing at the location right from the cross).

\section{Reaction Times}

A repeated-measures ANOVA was performed on median RTs (of correct responses only) with Compatibility (compatible vs. incompatible trials) and Sequence learning (Block 13 vs. the average of Blocks 12 and 14) as within-subjects factors. This analysis yielded a main effect of Compatibility, $F(1,41)=4.79, M S E=2330, p<.05$, with faster responses on compatible than on incompatible trials. There was also a main effect of Sequence learning, $F(1,41)=15.22, M S E=$ 2241, $p<.001$. The Compatibility $\times$ Sequence learning interaction, however, failed to reach significance, $F(1,41)$ $=2.74, M S E=1161, p=.11\left[M_{\text {comp }}=667 \mathrm{~ms}\right.$ (Block 
$13)$ and $630 \mathrm{~ms}$ (Blocks 12-14), $M_{\text {incomp }}=675 \mathrm{~ms}$ (Block 13) and $655 \mathrm{~ms}$ (Blocks 12-14)].

\section{Error Rates}

The repeated-measures ANOVA demonstrated a main effect of Compatibility, $F(1,41)=5.64, M S E=5.10, p<.05$, and a main effect of Sequence learning, $F(1,41)=7.00$, $M S E=2.35, p<.05$. Error rates were higher on incompatible trials than on compatible trials and in Block 13 than in Blocks 12-14. There was however no Compatibility $\times$ Sequence learning interaction, $F(1,41)<1\left[M_{\text {comp }}=2.17\right.$ (Block 13) and 1.40 (Blocks 12-14), $M_{\text {incomp }}=2.86$ (Block 13) and 2.37 (Blocks 12-14)].

In sum, manual motor effects do not seem to have influenced the perceptual learning effect observed in the current study.

\section{Discussion}

In the current experiment, we investigated perceptual learning of a visuospatial sequence in a paradigm in which eye movements were unnecessary. Participants were instructed to respond to the randomly varying identity of a target, while the sequence of locations was structured. Overt eye movements were avoided in two ways. First, we used a circular display in which target and distractors were placed around a fixation cross with the distance between the letter pairs and the fixation cross being substantially less than $1^{\circ}$ visual angle, namely $0.63^{\circ}$. This way, eye movements were unnecessary to locate the target. Second, stimulus duration was very short (100 ms) and insufficient to make eye movements. Despite these conditions to restrict saccades, reliable perceptual sequence learning was observed. Accordingly, the current findings show that perceptual learning can occur without overt eye movements. This suggests that perceptual learning of a visuospatial sequence can rely on learning of a sequence of covert attention shifts, as suggested by Remillard (2003).

Consequently, the current study adds support to the S-S learning view of sequence learning in the SRT task, stating that sequence learning can be based on associations between successive perceptual events. In other words, sequence learning is possible without the involvement of a motor component. The current experiment also revealed that perceptual sequence learning is quite immune to motor effects. First, it was not modulated by response repetition effects. This is not surprising, as there was no response repetition effect at all in the experiment, probably due to the RSI of $400 \mathrm{~ms}$. Previous research has shown that response repetition effects occur with short RSIs of $50 \mathrm{~ms}$, but usually disappear with longer RSIs (Soetens, 1998; Soetens, Boer, \& Hueting, 1985; Soetens, Melis, \& Notebaert, 2004). Even after practice with a sequence, no response repetition effect emerges when long RSIs are used (Soetens et al., 2004). Second, perceptual sequence learning did not seem to interact with stimulus-response compatibility effects. Earlier studies have demonstrated that motor sequence learning can reduce S-R compatibility effects (Deroost \& Soetens, 2006c; Koch, 2007). However, the current results suggest that the effect of sequence learning on compatibility effects is restricted to motor sequence learning, as perceptual sequence learning has no effect on compatibility effect sizes.

One issue that remains to be investigated is the role of explicit knowledge in perceptual sequence learning. Willingham (1999) found that participants who merely observed a sequence demonstrated sequence learning. However, when the participants displaying explicit knowledge were removed from the analyses, the perceptual learning effect disappeared. As we did not include an awareness measure in our study, we cannot rule out that some explicit knowledge existed in the experiment and supported the learning we found. However, our task was adapted from Remillard's paradigm $(2003,2009,2011)$, who repeatedly failed to find evidence for explicit knowledge. The fact that the visual angle used in the current experiment was substantially reduced compared to the original design and that the stimuli were only presented for $100 \mathrm{~ms}$ makes it rather unlikely that the learning effect was primarily governed by explicit knowledge.

In sum, the experiment provides one of the rare studies that explicitly aimed to examine the contribution of overt eye movements in a perceptual sequence learning design. Future studies using eye tracking are nevertheless necessary to substantiate our findings. For instance, in the current setup we cannot totally exclude the possibility that a number of participants still occasionally made eye movements during the experiment as the viewing distance was not fixated. We state that the distance between the fixation cross and the letter pairs was $0.63^{\circ}$ visual angle, but this is based on a screen distance of $55 \mathrm{~cm}$. Although we did not control for viewing distance by using a chin rest, it is important to keep in mind that the visual angle did not exceed $1^{\circ}$, as it is unlikely that participants approached the screen by less than $35 \mathrm{~cm}$. One could argue, however, that it still remains possible that participants moved their eyes toward the anticipated location during the RSI of $400 \mathrm{~ms}$ once they learned the location sequence, although the letter pairs themselves were only presented for $100 \mathrm{~ms}$. It should be noted that, even if this were the case, learning probably took place independent of the movements, as structured eye movements could only have started to occur after (part of) the sequence was learned. However, the eye movements may have contributed to the learning effect observed in our experiment. Indeed, the occurrence of micro-saccades during the RSI cannot be excluded using the current paradigm and should be further investigated using eye-tracking. If our findings can be confirmed in eye-tracking experiments, the current experiment provides a user-friendly behavioral paradigm that is easy to implement in the study of perceptual sequence learning.

For future research, it is important to clearly define what is meant by perceptual sequence learning. For instance, according to the premotor theory of attention, which recently gained more support (e.g., Belopolsky \& Theeuwes, 2009; Van der Stigchel \& Theeuwes, 2007), covert shifts of attention occur only after the eye movement has been programmed (Rizzolatti et al., 1987). Overt and covert attention shifts in this account are thus supposed to be ruled 
by the same mechanisms, but in case of a covert shift of attention the eye movement is blocked before being executed. At present, research cannot determine the programming of eye movements using visuospatial stimuli, so that, in fact, not a sequence of covert attention shifts but a sequence of aborted oculomotor programs may be learned in perceptual sequence learning studies, like the current. Consequently, this would mean that visuospatial sequence learning is never purely perceptual. Along this line, Remillard (2011) recently proposed that the neural base of visuospatial sequence learning might involve brain areas important for oculomotor movements, like the frontal eye field and the caudate nucleus.

To conclude, we have established a form of sequence learning when a perceptual sequence is imposed on the target's location and eye movements are made unnecessary. As participants seem to extract sequence knowledge under circumstances of sequenced manual responses, sequenced eye movements, and sequenced attention shifts, our findings support the notion of a strong learning mechanism that builds up knowledge in a highly flexible way, based on the sequenced information embedded within the environment. Future research should determine whether learning based on overt eye movements (see Albouy et al., 2006; Kinder et al., 2008; Marcus et al., 2006) and visuospatial sequence learning (with no or a minimal amount of saccades) is supported by the same mechanisms or alternatively relies on different systems.

\section{Acknowledgments}

The first author, Daphné Coomans, is a research fellow of the National Fund for Scientific Research of Flanders (Fonds voor Wetenschappelijk Onderzoek - Vlaanderen, Grant No. FWOTM506).

\section{References}

Abrams, R. A., \& Pratt, J. (2000). Oculocentric coding of inhibited eye movements to recently attended locations. Journal of Experimental Psychology: Human Perception and Performance, 26, 776-788.

Albouy, G., Ruby, P., Phillips, C., Luxen, A., Peigneux, P., \& Maquet, P. (2006). Implicit oculomotor sequence learning in humans: Time course of offline processing. Brain Research, 1090, 163-171.

Belopolsky, A. V., \& Theeuwes, J. (2009). When are attention and saccade preparation dissociated? Psychological Science, 20, 1340-1347.

Cleeremans, A., Destrebecqz, A., \& Boyer, M. (1998). Implicit learning: News from the front. Trends in Cognitive Sciences, $2,406-416$.

Clegg, B. A., DiGirolamo, G. J., \& Keele, S. W. (1998). Sequence learning. Trends in Cognitive Sciences, 2, 275-281.

Clohessy, A. B., Posner, M. I., \& Rothbart, M. K. (2001). Development of the functional visual field. Acta Psychologica, 106, 51-68.

Coomans, D., Deroost, N., Zeischka, P., \& Soetens, E. (2011). On the automaticity of pure perceptual sequence learning. Consciousness and Cognition, 20, 1460-1472.
Deroost, N., \& Soetens, E. (2006a). Perceptual or motor learning in SRT tasks with complex sequence structures. Psychological Research, 70, 88-102.

Deroost, N., \& Soetens, E. (2006b). Spatial processing and perceptual sequence learning in SRT tasks. Experimental Psychology, 53, 16-30.

Deroost, N., \& Soetens, E. (2006c). The role of response selection in sequence learning. Quarterly Journal of Experimental Psychology, 59, 449-456.

Eriksen, C. W., \& St. James, J. D. (1986). Visual attention within and around the field of focal attention: A zoom lens model. Perception \& Psychophysics, 40, 225-240.

Godijn, R., \& Pratt, J. (2002). Endogenous saccades are preceded by shifts of visual attention: Evidence from cross-saccadic priming effects. Acta Psychologica, 110, 83-102.

Kelly, S. W., \& Burton, A. M. (2001). Learning complex sequences: No role for observation? Psychological Research, $65,15-23$.

Kinder, A., Rolfs, M., \& Kliegl, R. (2008). Sequence learning at optimal stimulus-response mapping: Evidence from a serial reaction time task. Quarterly Journal of Experimental Psychology, 61, 203-209.

Koch, I. (2007). Anticipatory response control in motor sequence learning: Evidence from stimulus-response compatibility. Human Movement Science, 26, 257-274.

Marcus, D., Karatekin, C., \& Markiewicz, S. W. (2006). Oculomotor evidence of sequence learning on the serial reaction time task. Memory \& Cognition, 34, 420-432.

Mayr, U. (1996). Spatial attention and implicit sequence learning: Evidence for independent learning of spatial and nonspatial sequences. Journal of Experimental Psychology: Learning, Memory, and Cognition, 22, 350-364.

Nattkemper, D., \& Prinz, W. (1997). Stimulus and response anticipation in a serial reaction task. Psychological Research, 60, 98-112.

Posner, M. I. (1980). Orienting of attention. Quarterly Journal of Experimental Psychology, 32, 3-25.

Rayner, K. (1998). Eye movements in reading and information processing: 20 years of research. Psychological Bulletin, 124, 372-422.

Remillard, G. (2003). Pure perceptual-based sequence learning. Journal of Experimental Psychology: Learning, Memory, and Cognition, 29, 581-597.

Remillard, G. (2009). Pure perceptual-based sequence learning: A role for visuospatial attention. Journal of Experimental Psychology: Learning, Memory, and Cognition, 35, 528-541.

Remillard, G. (2011). Pure perceptual-based learning of second-, third, and fourth-order sequential probabilities. Psychological Research, 75, 307-323.

Rizzolatti, G., Riggio, L., Dascola, I., \& Ulmità, C. (1987). Reorienting attention across the horizontal and vertical meridians: Evidence in favor of a premotor theory of attention. Neuropsychologia, 25, 31-40.

Rüsseler, J., Münte, T. F., \& Rösler, F. (2002). Influence of stimulus distance in implicit learning of spatial and nonspatial event sequences. Perceptual and Motor Skills, 95, 973-987.

Schneider, W., Eschman, A., \& Zuccolotto, A. (2002). E-prime user's guide (Version 2.0). Pittsburgh, PA: Psychology Software Tools.

Schumacher, E. H., \& Schwarb, H. (2009). Parallel response selection disrupts sequence learning under dual-task conditions. Journal of Experimental Psychology: General, 138, 270-290.

Schwarb, H., \& Schumacher, E. H. (2009). Neural evidence of a role for spatial response selection in the learning of spatial sequences. Brain Research, 1247, 114-125.

Schwarb, H., \& Schumacher, E. H. (2010). Implicit sequence learning is represented by stimulus-response rules. Memory \& Cognition, 38, 677-688. 
Soetens, E. (1998). Localizing sequential effects in serial choice reaction time with the information reduction procedure. Journal of Experimental Psychology: Human Perception and Performance, 24, 547-568.

Soetens, E., Boer, L. C., \& Hueting, J. E. (1985). Expectancy or automatic facilitation? Separating sequential effects in twochoice reaction time. Journal of Experimental Psychology: Human Perception and Performance, 11, 598-616.

Soetens, E., Melis, A., \& Notebaert, W. (2004). Sequence learning and sequential effects. Psychological Research, 69, 124-137.

Van der Stigchel, S., \& Theeuwes, J. (2007). The relationship between covert and overt attention in endogenous cuing. Perception and Psychophysics, 69, 719-731.

Willingham, D. B. (1999). Implicit motor sequence learning is not purely perceptual. Memory \& Cognition, 27, 561-572.

Willingham, D. B., Nissen, M. J., \& Bullemer, P. (1989). On the development of procedural knowledge. Journal of Experimental Psychology: Learning, Memory, and Cognition, 15(6), 1047-1060.

Ziessler, M. (1998). Response-effect learning as a major component of implicit serial learning. Journal of Experimental Psychology: Learning, Memory, and Cognition, 24, 962-978.
Ziessler, M., \& Nattkemper, P. (2001). Learning of event sequences is based on response-effect learning: Further evidence from a serial reaction time task. Journal of Experimental Psychology: Learning, Memory, and Cognition, 27, 595-613.

Received August 10, 2011

Revision received January 12, 2012

Accepted January 12, 2012

Published online May 25, 2012

Daphné Coomans

Department of Cognitive Psychology

Vrije Universiteit Brussel

Pleinlaan 2

B-1050 Brussels

Belgium

Tel. +32 (0) 2/629.14.26

Fax +32 (0) 2/629.24.89

E-mail daphne.coomans@vub.ac.be 